\title{
Comparing acoustic and optical forces for biomedical research
}

\author{
Kishan Dholakia ${ }^{1,2, \dagger}$, Bruce W. Drinkwater ${ }^{3, \dagger}$ and Monika Ritsch-Marte ${ }^{4, \dagger}$ \\ ${ }^{1}$ SUPA, School of Physics and Astronomy, \\ University of St Andrews, UK. \\ 2 Department of Physics, College of Science, \\ Yonsei University, Seoul, South Korea. \\ 3 Faculty of Engineering, \\ University of Bristol, Bristol, UK. \\ 4 Institute of Biomedical Physics, \\ Medical University of Innsbruck, Innsbruck, Austria. \\ †e-mail: kd1@st-andrews.ac.uk; b.drinkwater@bristol.ac.uk; \\ monika.ritsch-marte@i-med.ac.at
}




\begin{abstract}
The application of acoustic and optical waves to exert non-contact forces on microscopic and mesoscopic objects has grown considerably in importance in the past few decades. Different physical principles govern the acoustic and optical forces, leading to diverse biomedical applications. Biocompatibility is crucial, and useful optical and acoustic forces can be applied in devices that maintain local heating to acceptable levels. Current acoustic and optical devices work on complementary length scales, with both modalities having useful capabilities at the scale of the cell. Optical devices also cover sub-cellular scales and acoustic devices super-cellular scales. This complementarity has led to the emergence of multi-mode manipulation, often with integrated imaging. In this Technical Review, we provide an overview of optical and acoustic forces, before comparing and contrasting the use of these modalities, or combinations thereof, in terms of sample manipulation and suitability for biomedical studies. We conclude with our perspective on the applications in which we expect to see notable developments in the near future.
\end{abstract}

\title{
KEY POINTS
}

- Acoustic and optical forces are governed by different physical principles, but both enable the application of non-contact forces to biomedically important objects such as cells and microorganisms.

- Acoustic and optical forces in the pico-Newton to nano-Newton range can be applied to a typical cell, with optical devices having capabilities extending below this scale and acoustic devices above.

- Biocompatibility cannot be assumed as both modalities can produce local heating; however, careful device design has led to many examples of biocompatible devices.

- Biomedical applications of optical and acoustic devices are rapidly increasing and include manipulation, patterning and mechanical probing, often combined with imaging.

- The number of applications is expected to increase, and we anticipate more examples of multimode or hybrid devices to emerge, increasingly sophisticated integration of imaging and the development of more versatile and fully reconfigurable manipulation systems. 


\section{[H1] INTRODUCTION}

Waves not only transport energy, but also convey momentum and, thus, can exert forces. When sound waves were recognized as elastic deformations propagating in a medium, it was intuitive to attribute mechanical momentum to them. Indeed, Lord Rayleigh did just that when he defined the acoustic radiation pressure. The concept of optical momentum in a beam of light propagating in vacuum, however, required Maxwell's electrodynamic description before it could be understood.

Despite the intrinsic differences between light and sound, the physical momentum they carry can in both cases be used to manipulate particles and objects across a range of length scales. Manipulation in this context refers to the non-contact confinement of the object in question. The exertion of calibrated forces induced externally by impinging wave fields has led to the creation of powerful tools for biomedical research. These tools enable or facilitate a wealth of operations or assays, from quantitative force measurements to the contact-free immobilization of objects for imaging and spectroscopy.

In this Technical Review, we describe how optical and acoustic forces have come to the fore over the past two decades and how they offer complementary routes for non-contact manipulation, yet may also be used together on occasion. By comparing and contrasting the forces that these waves exert, we illustrate the applications in which they are best employed. Examples of acoustic and optical forces operating in tandem (Fig.1) are also described. This Technical Review mainly focuses on 'tweezers', which we interpret in the broad sense of a converging force field that traps a particle. However, we also briefly describe related forceapplication devices, which might be better described as deflectors, with an emphasis on manipulation in liquids. We conclude with our perspective on the future directions of the field.

\section{[H1] OPTICAL FORCES}

Optical forces arise when objects absorb, refract or scatter light and momentum is transferred. For levels of optical power that do not damage cells, these forces are very small, typically on the order of pico-Newtons. This force may be negligible in the macroscopic world, but this is not the case for microscopic (sub-millimetre) particles. Indeed, with the 
Nobel Prize in Physics having been awarded to this field in 2018, these small forces are now being fully recognized for the wide-spread impact they have had on biomedical research. In 1970, Arthur Ashkin first reported the optical trapping of a microsphere placed within a laser field [1] — optical trapping was born. This first optical trapping concept was based on a counter-propagating beam geometry and did not require tightly focused light fields.

The breakthrough in optical manipulation came in 1986, when Ashkin and colleagues first introduced what are today aptly called optical tweezers. With a tightly focused laser, a single laser beam can be used to trap, hold and move a microscopic particle, provided that its (average) refractive index is larger than that of its surroundings [2].

The forces at work in the optical traps discussed herein can be derived from Maxwell's theory applied to the interaction of dielectric particles with electromagnetic waves in the visible or near-infrared. Starting with the general Maxwell stress tensor, one can derive the following far-field expression for the optical force on a dielectric particle in a medium of refractive index $n_{\mathrm{m}}$ and dielectric permittivity $\varepsilon_{\mathrm{m}}$ in terms of the incident and scattered electric $(E)$ and magnetic $(B)$ fields, $E_{\mathrm{i}}, B_{\mathrm{i}}$ and $E_{\mathrm{s}}, B_{\mathrm{s}}$, respectively,

$$
\begin{array}{r}
\mathbf{F}_{\mathrm{opt}}=-\frac{\varepsilon_{\mathrm{m}}}{4} r^{2} \int_{\Omega} \mathrm{d} \Omega \mathbf{e}_{\mathrm{r}}\left[\left|\mathbf{E}_{\mathrm{s}}\right|^{2}+\frac{c^{2}}{n_{\mathrm{m}}^{2}}\left|\mathbf{B}_{\mathrm{s}}\right|^{2}\right. \\
\left.+2 \Re\left(\mathbf{E}_{\mathrm{i}} \cdot \mathbf{E}_{\mathrm{s}}^{*}+\frac{c^{2}}{n_{\mathrm{m}}^{2}} \mathbf{B}_{\mathrm{i}} \cdot \mathbf{B}_{\mathrm{s}}^{*}\right)\right] .
\end{array}
$$

Here, $c$ is the speed of light in vacuum, $\mathbf{e}_{\mathrm{r}}$ is the radial unit vector, and the integration is over the full solid angle $\Omega$ of a sphere with a large radius $r$ around the particle. The force given in Eq.(1) describes the total force acting on the particle and can be understood in terms of momentum conservation. For an explicit yet concise treatment of the theory of optical forces, including the derivation of the equations in this section, we refer readers to Ref. [3].

In principle, the above explicit expression can be used to calculate the forces for arbitrary input fields and particles of arbitrary shape. An exception are soft deformable particles that change shape under the influence of the optical force, affecting the scattered and refracted light, for which a self-consistent treatment of the optical and elastic forces is required [4, 5]. The challenge in evaluating the above expression lies in the calculation of the scattered light. For spherical particles, the generalized Lorenz-Mie theory $[6,7]$ can be used to calculate the force, whereas numerical simulations can be used for more complex cases $[8,9]$. 
It is illustrative (and of particular relevance in biomedical applications) to study the relatively simple case of a particle that can be described by an electric dipole in a monochromatic field. This can be an atomic or molecular dipole, or a sizeable dielectric object in the (sub-)micrometre range that consists of many atomic or molecular dipoles. The dipole approximation holds particularly well for 'Rayleigh scatterers' that satisfy the condition $k a<1$, where $a$ is the particle size (for example, the radius of a bead) and $k$ is the optical wavenumber in the medium. The Clausius-Mosotti (or Lorentz-Lorenz) relation builds a bridge between microscopic parameters (such as the number density, $N$, of molecules per volume and the molecular polarizability, $\alpha_{\mathrm{m}}$ ) and bulk material parameters (such as the refractive index, $n$ ), through the relation $\frac{n^{2}-1}{n^{2}+2}=\frac{N \alpha_{m}}{3 \varepsilon_{0}}$ (where $\varepsilon_{0}$ is the vacuum permittivity).

The time-averaged force on a small dielectric particle that can be described by an effective dipole is given by [3]

$$
\mathbf{F}_{\mathrm{d}}(\mathbf{r})=\frac{\alpha_{\mathrm{r}}}{4} \nabla\left|\mathbf{E}_{\mathrm{i}}(\mathbf{r})\right|^{2}+\frac{\sigma}{c} \mathbf{S}_{\mathrm{i}}(\mathbf{r})-\frac{\sigma c}{2} \nabla \times \mathbf{s}_{\mathrm{i}}(\mathbf{r})
$$

where $\alpha_{\mathrm{r}}$ denotes the real part of the particle's effective polarizability. $\sigma$ represents the extinction cross section, which includes the cross section for absorption and for scattering and is proportional to the imaginary part of the particle's polarizability. In most cases covered in this Technical Review, the particle does not absorb energy from the input field, and thus scattering dominates the extinction cross section. Note that in contrast to laser cooling of atomic systems in vacuum [10], which uses incoherent scattering by spontaneous emission, here, we mean only coherent scattering. $\mathbf{S}_{\mathrm{i}}$ is the time-averaged Poynting vector, and $\mathbf{s}_{\mathrm{i}}=\left\langle i \frac{\varepsilon_{0}}{2 \omega} \mathbf{E}_{\mathrm{i}} \times \mathbf{E}_{\mathrm{i}}^{*}\right\rangle$ is the time-averaged spin density (where $\omega$ is the angular frequency of the input field).

There are three distinctive terms in the expression for the force on the dipole in Eq.(2). The first term can be rewritten as $\mathbf{F}_{\text {grad }}(\mathbf{r})=\frac{\alpha_{\mathrm{r}}}{2 c \varepsilon_{0}} \nabla I_{\mathrm{i}}(\mathbf{r})$ in terms of the intensity $I_{\mathrm{i}}=$ $\frac{1}{2} c \varepsilon_{0}\left|E_{\mathrm{i}}\right|^{2}$ ( Fig.2a)), which acts as an effective potential energy landscape. Therefore, the first force term is usually called the gradient force (Fig.2b). Particles with a refractive index larger than that of their surroundings, and thus a positive polarizability, are 'high-field seekers', which are drawn into the laser spot by a force that is proportional to the optical intensity gradient. The trapping of low-index particles immersed in a fluid, such as a gas bubble in a liquid, which are driven out of the high-intensity regions, requires a different strategy of creating a 'dark' trap [11]. 
The second term also contributes in typical biophotonics applications. This term is proportional to the imaginary part of the complex polarizability responsible for out-ofphase scattering of light by the small dielectric object. For a dipole scattering light, this (non-conservative) force points in the direction of the local energy flow of the incoming light (Fig.2c). In an optical tweezers system with a focused beam, the effect of this force is to displace the stable trapping position slightly 'downstream' from the laser focus. This force contribution — which prevails in homogeneous fields - is often called the radiation pressure force.

For anisotropic scattering from more extended particles $(a \geq \lambda$, where $\lambda$ is the wavelength of the incident light), which cannot be accurately described by an effective dipole, the radiation pressure force can push the particle in directions that differ from the direction of the incident light beam. In particular, asymmetric particles under complicated illumination set-ups can display counter-intuitive behaviour, such as the 'tractor-beam' effect $[12,13]$. For large dielectric particles, the dipole approximation fails and it is necessary to revert to general expressions such as Eq.(1) to determine the redistribution of optical momentum by the particle. However, for particles much larger than the wavelength, the description can again be simplified, as the principles of geometrical optics become applicable, and thus scattering 'turns into' refraction and reflection.

At first, it may seem plausible that increasing the relative refractive index of the particle should facilitate optical trapping, but the situation is more complicated. For a large spherical particle in the geometrical optics regime, there are some limiting cases: index-matched spheres cannot be trapped, and particles with a very high relative refractive index experience strong back-reflection and are thus strongly pushed forward. But predicting the refractive index and size regimes in which the gradient force is sufficiently strong to ensure stable trapping of a small sphere generally requires a detailed analysis. In particular, when the size of the particle is similar to the optical wavelength, intricate patterns of stable trapping regions form depending on the particle radius, the relative refractive index and the numerical aperture (NA) of the trapping beam, which can be explained in terms of Mie resonances [7].

The third term in Eq.(2) — the 'spin-curl force' — is related to polarization gradients that may be present in the input field. This force does not have a prominent role in the optical manipulation of biomedical samples, and thus is not further considered in this Technical Review. As this term is intrinsically associated with the polarization of the optical wave, it 
does not have a direct counterpart in acoustic waves. However, acoustic waves can possess orbital angular momentum $[14,15]$ and, as we discuss below, several acoustic trapping devices are based on vortex beams with orbital angular momentum.

Finally, optical fields may feature more exotic forms of spin-orbit coupling and other forces that have no counterparts in the acoustic domain and are thus not considered here.

The single-beam laser trap formed by a tightly focused laser beam proved to be a highly useful experimental tool, especially when used in combination with a wavefront shaper to flexibly steer the position of the laser spot in real time in three dimensions. With devices generally known as holographic optical tweezers [16], it is possible to manipulate living biological cells in liquids. By consideration of the appropriate internal resonances, the physical principles underlying optical tweezers can be extended to trap, for example, atoms or Bose-Einstein condensates in vacuum.

Benefits of the focused single-beam trap are its ease of assembly and usability. Yet, for larger (and thus heavier) objects that require a higher peak power, counter-propagating beam configurations are preferable, as they support trapping in low(er) intensity regions. For example, using this beam configuration, a particle can be confined to a region between two laser spots by means of balanced radiation pressure forces [17]. This configuration reduces the potential for photodamage and also supports larger interaction volumes.

Force calibration is important, as it is not always possible to estimate the forces from first principles in a complicated set-up. Many methods for force calibration in optical manipulation have been explored [3], including direct all-optical approaches that determine the force directly from the change in optical momentum brought about by the particle [18, 19]. Calibration turned optical traps into quantitative measurement devices that have revolutionized single-molecule biophysics owing to their ability for repeatable, calibrated force measurements in the pico-Newton regime. This includes force measurements in active singlemolecule systems, ranging from DNA strands [20], even with single-base-pair precision [21], to single proteins and receptor molecules [22, 23]. For such studies, one can simultaneously maximize the trap stiffness while minimizing errors in the force measurements by using attached beads, which serve as trapping handles, with an optimum nominal bead size matched to the beam waist (that is, $250-500 \mathrm{~nm}$ ), as this is an operating point that is insensitive to (inevitable) variations in bead size [24]. 


\section{[H1] ACOUSTIC FORCES}

If we now turn to acoustics, how do we understand the interaction of a sound wave with a particle? In 1971, around the same time as optical trapping was discovered but long before the realization of dedicated acoustic traps, it was noticed that a standing ultrasound wave can exert a force that stops blood cells in blood vessels [25].

Just as with the optical counterpart, acoustic radiation forces originate from the scattering and absorption of acoustic waves, and hence there are gradient and scattering force terms that are analogous to the optical force contributions. But, as we discuss below, in typical applications, the size of the manipulated sample is considerably smaller than the acoustic wavelength. This holds for the two most common configurations: the use of tens of kilohertz in air for the manipulation of objects in the 100-1,000 $\mu \mathrm{m}$ range, as well as at megahertz in liquids for the manipulation of objects in the 1-100 $\mu \mathrm{m}$ range.

In the acoustic case, the forces are derived from the dynamic Navier-Stokes equations for fluid mechanics. These equations describe acoustic radiation forces, as well as the transfer of momentum to the surrounding fluid, which induces fluid flow, an effect known as acoustic streaming. Hence, the particle experiences not only radiation force components due to pressure gradients $\left(\mathbf{F}_{\text {grad }}\right)$, scattering $\left(\mathbf{F}_{\text {scat }}\right)$ and absorption within the particle $\left(\mathbf{F}_{\text {abs }}\right)$, but also drag forces due to the streaming $\left(\mathbf{F}_{\text {str }}\right)$,

$$
\mathbf{F}_{\mathrm{ac}}=\mathbf{F}_{\text {grad }}+\mathbf{F}_{\mathrm{scat}}+\mathbf{F}_{\mathrm{abs}}+\mathbf{F}_{\mathrm{str}}
$$

Perturbation analysis can be used to derive formulae for the terms in Eq.(3). In such analyses, the harmonically oscillating standing pressure and velocity in sound waves average to zero, and the lowest non-vanishing order of the time-averaged fields (pressure $(p)$, density $(\rho)$ and velocity $(v))$ that contribute to the acoustic forces are second order. In many practical cases of importance in biomedical applications, the gradient forces dominate and elegant expressions can be obtained from the perturbation analyses. Of particular importance here is the regime in which the size of the particle is considerably smaller than the acoustic wavelength in the fluid, named the Rayleigh regime in both optics and acoustics. In this regime, the Gor'kov potential theory [26], developed in 1961, can be used to calculate the gradient force. Although Gor'kov assumed a frictionless fluid and spherical fluid particles, his potential theory has been found to be applicable to many microfluidic acoustic radiation 
force devices [27].

$$
\mathbf{F}_{\text {grad }}=-\nabla U
$$

where the Gor'kov potential, $U$, is given by

$$
U=\frac{4 \pi a^{3}}{3}\left[f_{1} \frac{1}{2} \kappa_{0}\left\langle p^{2}\right\rangle-f_{2} \frac{3}{4} \rho_{0}\left\langle v^{2}\right\rangle\right] .
$$

Here, $\left\langle p^{2}\right\rangle$ and $\left\langle v^{2}\right\rangle$ are the time-averaged acoustic pressure and velocity, respectively, $a$ is the sphere radius, and $\kappa_{0}$ and $\rho_{0}$ are the compressibility and density of the host fluid, respectively. The material-dependent pre-factors are given by $f_{1}=1-\kappa_{P} / \kappa_{0}$ and $f_{2}=$ $2\left(\rho_{P}-\rho_{0}\right) /\left(2 \rho_{P}+\rho_{0}\right)$, where the subscript $P$ denotes a property of the particle. In Eq.(5), the first term, proportional to $\left\langle p^{2}\right\rangle$, corresponds to a monopole contribution due to radial oscillations, and the second term, proportional to $\left\langle v^{2}\right\rangle$, represents a dipole contribution due to linear oscillations of the particle [28]. The monopole contribution is only relevant for particles with a compressibility that is different from that of the surrounding liquid.

The patterning of the particles inside the acoustic pressure landscape depends on the acoustic contrast parameters of the particle and the surrounding fluid, namely $f_{1}$ and $f_{2}$ in Eq.(5). In many cases in biomedical research, however, the particles are small relative to the wavelength and have a higher density than the host medium (typically water), meaning that they accumulate at the low-pressure regions known as nodes.

For the very simplest form of the acoustic field, a 1D sinusoidal standing wave of sound pressure $p=p_{0} \cos k x \sin \omega t$ (where $\omega$ is the angular frequency, and $t$ is time), Eq.(4) can be simplified to

$$
F_{\text {grad }}=-4 \pi \phi k E a^{3} \sin (2 k x)
$$

where $E=p^{2} / 4 \rho_{0} c_{0}^{2}$ (where $c_{0}$ is the speed of sound in the host medium) is the acoustic energy density, $\phi=1 / 3 f_{1}+1 / 2 f_{2}$ is often referred to as the acoustic contrast factor and $k=2 \pi / \lambda$ is the acoustic wavenumber in the host fluid [27]. Eq.(6) is the governing equation for many of the planar channel resonator devices used widely in lab-on-a-chip applications such as filtering [29]. One-dimensional-like fields have also been used for cell patterning applications in which the cells are formed into lines along the nodes [30, 31].

The acoustic potential force (Fig.3) is analogous to the gradient force in optical tweezers. Single-beam gradient-force acoustic tweezers have been realized in air [32] and in water [3336]. 
There is, however, an important difference between acoustic tweezers and optical tweezers. In optical tweezers, the refractive index of the sample is usually higher than in the surroundings, which means that the gradient forces push the sample into a maximum of the electric field. By contrast, for acoustic tweezers (in particular for trapping of any solid or liquid in air and for most cells in water-based biological media) the property contrast is usually the other way round, and the sample is expelled from high-pressure (antinode) regions and drawn into a pressure minimum (node). Therefore, it is necessary to use confining trap configurations that resemble dark optical traps, such as by using Laguerre-Gauss beams to trap low-index particles [11]. In both modalities, vortex beams carrying orbital angular momentum are employed for this kind of confinement [37-41].

As can be seen from Eq.(3), the acoustic potential is one part of the full expression, which also contains force terms due to absorption and scattering, the latter being the acoustic counterpart of the non-conservative optical scattering force. Corrections that, for example, take the viscosity of the fluid and heating effects into account [42] are available but usually remain small in typical microfluidic applications. Therefore, at least for particles smaller than the wavelength, an effective potential can be used to derive the dominant forces [26, 27].

For spherical particles experiencing a 2D ultrasonic standing wave in a rectangular cavity, the behaviour can be simulated in detail and force equations derived beyond the applicability of Gor'kov theory [43]. Analogous to the situation in optics, in the regime in which the particle's size is similar to the wavelength, resonances in the particle lead to variation in the sign and strength of the force, dependent on the particle's size relative to the wavelength and the acoustic contrast parameters of mass density and compressibility. For example, polystyrene spheres of size $20-90 \mu \mathrm{m}$ in a $6.5 \mathrm{MHz}$ field in water stably settle into the pressure nodes of the standing ultrasound waves. By contrast, beads of the same material that are more than 10 times smaller accumulate in the midpoints between nodes and antinodes. It has also been demonstrated both numerically and theoretically that, owing to the contrast effect, similarly sized polystyrene spheres and osmotically swollen red blood cells can experience dramatically different force landscapes in the same standing-wave field [43]. Acoustic microbeam devices have been developed that operate at $>50 \mathrm{MHz}$ in this Mie regime [44].

The so-called secondary radiation or Bjerknes forces [45] arise when the scattered sound waves induce non-negligible interactions between neighbouring particles. These forces are called secondary forces because they arise from second-order scattering, that is, from the 
re-scattering of acoustic waves between the particles, which leads to an effective particleparticle momentum exchange or force. The corresponding optical effect, whereby two closely separated trapped particles influence each other by optical scattering, is called optical binding $[46,47]$.

Although acoustic secondary forces are typically small (scaling with $d^{-4}$, where $d$ is the inter-particle separation), they lead to observable effects, such as increased particle agglomeration, when particles are in close proximity. This effect has been used to attract and bind small extracellular vesicles $(\sim 100 \mathrm{~nm})$ to large particles $(12 \mu \mathrm{m})$ seeded in a standing-wave trap [48].

Various devices have been developed to excite the ultrasonic waves required for the application of acoustic forces. The most common approaches are excitation of bulk acoustic waves in a fluid-filled chamber or the use of surface acoustic waves [49]. A surface acoustic wave is an acoustic wave travelling along the surface of an elastic or piezoelectric substrate. This wave decays exponentially with distance from the surface and thus resembles the optical phenomenon of exponentially decaying 'evanescent' fields extending into a material where the electromagnetic wave cannot penetrate. Because of the ease with which they can be integrated into lab-on-a-chip systems, surface-acoustic-wave devices with 1D force fields (that is, governed by Eq.(6)) have been widely used in a range of filtering, patterning and actuation applications [50-52]. For both bulk-acoustic-wave and surface-acoustic-wave devices, operating frequencies are typically in the range 1-100 MHz. Lower frequencies result in a wavelength that is too long to be useful, and higher frequencies are limited by the rapid increase in absorption. As wavelengths are thus in the range $15-1,500 \mu \mathrm{m}$, it is apparent why the acoustic manipulation of cells is nearly always in the Rayleigh regime, but for microorganisms, it can be in the Mie regime. Alternative excitation schemes that involve low-frequency vibrations have also been explored for low-cost devices [53, 54].

In contrast to optical trapping, acoustic trapping is accompanied by acoustic streaming of the fluid. The streaming mechanisms involve either momentum transfer to the fluid or boundary effects, with the latter making streaming highly dependent on the device geometry [55]. Streaming is usually unwanted, and devices are preferentially operated in a regime wherein gradient forces dominate and streaming-induced drag forces are small. The common approach is to design devices with geometries and acoustic intensities such that the acoustic radiation force is larger than the streaming-induced Stokes force. A critical particle size for 
which both forces are of equal strength can be found [56]. As streaming is a weak nonlinear effect, it can be decoupled and accurately modelled by the application of body forces to a fluid domain, negating the need to model the fully coupled dynamic Navier-Stokes equations [57]. However, streaming can sometimes be exploited to, for example, facilitate mixing, and this is typically achieved in travelling wave fields [58].

For more detailed insight into acoustic manipulation, we refer readers to a series of articles introduced in ref [59].

\section{[H1] OPTICAL VERSUS ACOUSTIC MANIPULATION}

In view of the different physics governing optical and acoustic waves, it is somewhat surprising that very similar tools exist for the mechanical manipulation of microscopic particles (such as biological cells) in water. Concerning acoustic manipulation, air devices were developed in the 1980s and 1990s, before the development of devices that operate in liquids. However, in this Technical Review, we focus on the more recent liquid-based acoustic trapping devices that are closest to optical trapping and their biomedical applications in liquid environments, such as lab-on-a-chip applications. Apart from trapping, numerous efforts have been made towards 'acoustophoretic' devices, for acoustically induced manipulation and separation of microfluidic particles [29, 60].

In both optics and acoustics, the size of the particle has an important role in determining the radiation forces. However, an additional contrast is required between the properties of the particle and those of the surrounding liquid in order to apply manipulation forces strong enough to hold or move the particle. As the underlying physical principles are different, the optical and acoustic contrast parameters are fundamentally different, which is advantageous as it increases the number of options for manipulation in a given situation. In optical trapping, the most important contrast parameter is the dielectric polarizability, that is, the relative refractive indices of the particle and the medium. For acoustic trapping, the mass density and compressibility of the particle relative to the host fluid govern the acoustic contrast. In many biomedically relevant scenarios, such as the manipulation of single

cells or microorganisms, sufficient acoustic contrast exists (typically due to small density differences between cells and water) such that acoustic tweezers that work analogously to optical tweezers can be realized [34]. 
In the following, we assess the advantages and limitations of the two trapping modalities and compare them with respect to practical considerations.

\section{[H2] Reconfigurability}

A major benefit of optical trapping is the ease and the flexibility with which the traps can be reconfigured. Holographic optical tweezers allow multiple optical traps to be efficiently and quickly adapted or adjusted in three dimensions [16]. Considerable progress in this respect has also been made in acoustics, and acoustic traps have become more dynamic [61, 62], using, for example, phased arrays or multibeam devices. Recently, the acoustic analogue of holographic optical tweezers, holographic acoustic tweezers, was realized [63]. The acoustic system uses two phased arrays with 256 emitters that operate at $40 \mathrm{kHz}$ in air and was able to individually and simultaneously manipulate up to 25 particles of millimetre-size. The required pressure node configurations for the particles were created using an iterative algorithm based on the Gerchberg-Saxton algorithm, which is widely used in optical holography and in holographic optical tweezers.

Owing to the smaller scale of optical wavelengths, it is, however, difficult to surpass optical tweezers in regard to shaping the trapping fields on a local scale or steering individual particles in three dimensions. Nevertheless, although a standing ultrasonic wave in a planar geometry may be less reconfigurable, it does offer the possibility for the parallel manipulation of thousands of particles simultaneously [64].

Parallels between optical and acoustic fields can be exploited to reveal physical insight; for example, the acoustic tractor beam followed directly from its optical equivalent $[65,66]$.

\section{[H2] Range of trapping sizes}

Figure 4 provides an overview of the acoustic and optical trapping regimes. Optical manipulation is feasible for a broad range of particle sizes [3, 67], ranging from biological specimens of $\sim 1-100 \mu \mathrm{m}$ in water and nanoparticles, down to individual molecules and cold atoms in vacuum [68]. To reach the nanoscale, plasmonic resonances and optical near-field effects can be used [69,70], which increase the optical gradient force and, thus, facilitates trapping of such small particles. 
As in optical trapping, the sizes of particles or objects that can be handled by acoustic forces span a wide range, from centimetres and millimetres down to the nanoscale, with the latter realized with porous metal nanoparticles of high compressibility [71].

We now compare optical and acoustic gradient forces using a specific example, namely polystyrene beads of radius $a=1 \mu \mathrm{m}$ and $a=10 \mu \mathrm{m}$ in water. Using a $10 \mathrm{~mW}$ laser beam with a wavelength of $1 \mu \mathrm{m}$ focused to a spot by a lens with a NA of 1.2 (that is, with a peak intensity of $\sim 10^{6} \mathrm{~W} \mathrm{~cm}^{-2}$ ), the resulting maximal lateral force on the smaller bead is $\sim 26$ $\mathrm{pN}$, with a stable lateral trapping range of $\pm 0.4 \mu \mathrm{m}$ and a lateral trapping stiffness of 55-80 $\mathrm{pN} \mu \mathrm{m}^{-1}$. Increasing the bead size to $a=10 \mu \mathrm{m}$ only slightly increases the maximal force to $\sim 35 \mathrm{pN}$, in a trapping range of $\pm 5 \mu \mathrm{m}$, but at a considerably lower trapping stiffness of

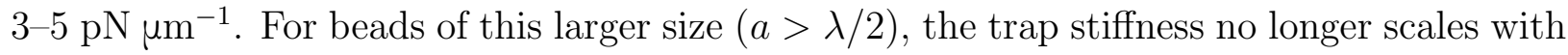
$a^{3}$, and there are strong deviations from the harmonic behaviour (the given number holds close to the trap centre where the trap is linear).

For this same scenario, using Eq.(6), we obtain an order of magnitude estimate of $\sim 1$ $\mathrm{nN}$ for the acoustic gradient force on a particle of $a=10 \mu \mathrm{m}$ in water within a $1 \mathrm{MHz} 1 \mathrm{D}$ standing-wave field of amplitude $1 \mathrm{MPa}$ (or an intensity of $67 \mathrm{~W} \mathrm{~cm}^{-2}$ ) and an estimate

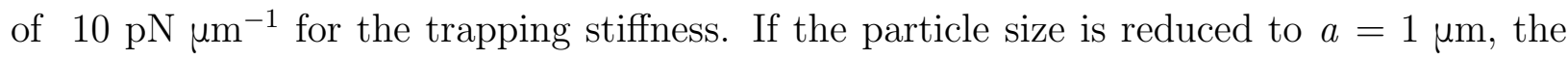
force becomes $\sim 1 \mathrm{pN}$, which would typically be less than the streaming force $\left(\mathbf{F}_{\text {str }}\right)$, making controlled manipulation of this particle size and frequency combination challenging. The conclusion is that optical forces operate on a smaller scale and thus lead to a higher degree of particle localization, whereas acoustic forces are greater for larger particles.

In terms of biocompatibility, which is discussed in more detail below, it is clear that acoustic manipulation is the better option for dense particles larger than $100 \mu \mathrm{m}$ in diameter. Acoustic tweezers can achieve forces as strong as micro-Newtons from intensity levels on the order of $50 \mathrm{~W} \mathrm{~cm}^{-2}$ on particles with sizes of $\sim 200 \mu \mathrm{m}$ Ref.[34]. By contrast, intensities of $10^{6}-10^{7} \mathrm{~W} \mathrm{~cm}^{-2}$ are typically used by single-beam optical tweezers to generate forces in the pico-Newton regime. The force achievable for a given invested power is an important figure of merit, and in this regard, acoustics (at least in principle) outperforms optics by many orders of magnitude.

Concerning the achievable trapping forces, a basic argument can be given for why the forces are much larger in acoustics than in optics. The momentum contained in a wave can be written as energy over phase velocity, and thus the force scales as $P / c$, where $P$ 
denotes the power in the wave, and $c$ is the phase velocity. Therefore, the difference in the transferable forces in optics and in acoustics can, in principle, be explained by the ratio of the speed of light to the speed of sound. But, one should not forget that optical tweezers and acoustic tweezers operate at different scales in terms of the relative sizes of the object and wavelength. Optically, all of the incident power is effectively focused by a high-NA lens into the particle volume, whereas a particle trapped by acoustic tweezers is typically much smaller than the acoustic wavelength and thus only inefficiently experiences the impinging power. This explains why the difference of force regimes for optical tweezers and acoustic tweezers is less than expected based on the ratio of the respective $P / c$ values.

To consider a specific example, we assume an optical or acoustic input power of $100 \mathrm{~mW}$ is applied to a 10- $\mu \mathrm{m}$ diameter particle in water using either a 1,064 nm trapping laser with a wavelength of $\lambda_{\mathrm{o}} \approx 800 \mathrm{~nm}$ in the chamber or a $1 \mathrm{MHz}$ ultrasound wave with a wavelength of $\lambda_{\mathrm{a}} \approx 1.4 \mathrm{~mm}$. From the ratio of the speed of light to the speed of sound, $c_{\mathrm{o}} / c_{\mathrm{a}} \approx 10^{5}$, we might expect to achieve a force from the acoustic power that is five orders of magnitude larger than that from the optical power. However, the volume of the particle is $\sim 10^{3} \mu \mathrm{m}^{3}$, which, for the optical case, is $\sim 8,000$ times the focal volume of $\lambda_{\mathrm{o}}^{3}$, guaranteeing efficient coupling to the incident power. By contrast, in the acoustic case, the particle experiences only a fraction, $\sim 10^{-6}$, of the power in the volume of $\lambda_{\mathrm{a}}^{3}$ in the focus. This argument is, of course, too simplistic and not suitable for the practical calculation of forces, but it does give valuable physical insight to demonstrate that it is not strictly valid to estimate the achievable acoustic and optical forces for the same power level by simply calculating the value of $P / c$.

Although both optical and acoustic forces are generally associated with particle confinement, they can also be used for particle deflection, selection or sorting. This is particularly the case in microfluidic environments at low Reynolds numbers. In active sorting with optical or acoustic forces, a sample can be interrogated through mechanisms of fluorescence or scattering, and subsequently the optical or acoustic field is used to deflect the sample of interest to an appropriate reservoir $[29,58,60,72]$ downstream to deflect or sort the object away from the main flow region. In biomedicine, this technique could be exploited for small sample volumes in microfluidic versions of flow cytometers. However, throughput is an issue, particularly for optically enabled sorters, owing to the weak forces.

For passive sorting, separation requires a difference in the affinity of the particles to an 
underlying potential energy landscape. In acoustic microchannel flow-through devices, the differing affinities are the result of differences in the particle size and acoustic contrast parameters, leading to a range of trajectory-based sorting configurations [60]. In analogous optical devices, Brownian motion and characteristic depths of the potential energy landscape (which should be several $k_{\mathrm{B}} T$ deep, where $k_{\mathrm{B}}$ is the Boltzmann constant and $T$ is temperature) lead to differing particle trajectories based on parameters such as size or refractive index [73]. This approach is label-free, and a very high sensitivity can be achieved though particle extraction although jamming may be an issue.

\section{[H2] Biocompatibility}

The biocompatibility of a tool is essential for its application in biomedical research. In this respect, optical tweezers have proved suitable for a multitude of applications. Nevertheless, there is a limit on the sample size, as the laser power necessary to hold larger and, thus, heavier loads requires an increase in laser power. Crucially, all this power is focused on the cell in simple single-beam optical tweezers. Residual absorption, which always occurs, will thus lead to heating and subsequent 'opticution', a term coined by Ashkin. This is the main reason why near-infrared lasers (for example, at a wavelength of 1,064 nm) are typically used as a compromise between absorption by pigments in the visible region and increasing absorption by water for wavelengths into the near-infrared [74]. Longer wavelengths also tend to mitigate the risk of phototoxic effects.

The limit on the size of the biological sample can be substantially increased to $\sim 100$ $\mu \mathrm{m}$ by using trapping configurations with counter-propagating beams, in which trapping is achieved by balanced radiation pressure forces at a point between two laser spots [17, 75]. With such 'macro-tweezers', it is possible to manipulate large microorganisms, such as Euglena gracilis protists, which are strong swimmers, to stall their motion and to force them

on an arbitrary trajectory, without causing apparent harm. The large working distance of several millimetres of this trap enables counter-measures against heating, such as by active cooling, should it be necessary when pushing the limits.

Nevertheless, the absorption limit is reached on going to even larger organisms - they cannot be held optically without inducing heating or other photodamage [76, 77]. The practical size limit for optical trapping with a near-infrared beam depends on the absorption 
coefficient of the sample, but even for very transparent samples, the limit is eventually reached for sizes beyond $\sim 80-100 \mu \mathrm{m}$.

Although acoustic tweezers may not have a power advantage over optical tweezers of five orders of magnitude, they still have an advantage in this respect, enabling, for example, the levitation of entire living organisms, such as a Caenorhabditis elegans nematode [78]. Possible issues with acoustic manipulation at high pressure and power levels are heating and cavitation. It is sometimes argued that acoustic tweezers are harmless, as they use irradiation levels comparable to those of ultrasonic imaging devices, the non-invasiveness of which is extremely well documented. But the intensity of acoustic traps in water (for example, $\sim 42 \mathrm{~W} \mathrm{~cm}^{-2}$ in Ref. [34]) is often higher than the intensity levels of medical diagnostic ultrasound, for which it is recommended to stay well below $1 \mathrm{~W} \mathrm{~cm}^{-2}$. Moreover, it is not clear that the conclusions drawn from sonography can be generalized to acoustic trapping, because medical imaging devices use pulsed operation, which is less prone to heating the tissue. Moreover, effects on individual cells and on different types of cells in an in vitro microfluidic cell assay may be different, and will depend on where the cell is located within the field, for example, in a node or an antinode of a standing acoustic wave or a propagating wave.

In acoustofluidic lab-on-a-chip devices, considerable heating may also occur, necessitating temperature control. Typically, the temperature rise is not only caused by absorption in the liquid or the sample, but also by heat losses in the piezoelectric transducer element itself or in thin glue layers used in the construction of the devices [79]. Heating is especially important for small-volume (approximately tens of microlitres) microfluidic chambers in which active cooling, such as through Peltier plate devices, may be required [80].

Cavitation is a highly destructive effect that can be used, for example, for cleaning. But as this effect scales with the inverse square root of the ultrasound frequency $1 / \sqrt{f}$ (ref.[81]), the formation of cavitation bubbles is unlikely for the frequencies of a few megahertz that are typically used in acoustofluidics. In other words, in typical trapping situations, cavitation is easy to avoid and is more of a device design consideration than a problem in real devices. If the fluid already contains gas-filled bubbles, the acoustic pressure waves cause the bubbles to oscillate. Oscillating microbubbles can apply large shear forces to biological objects to induce a number of bio-effects on microorganisms and in blood vessels and have found several biomedical applications, such as enhanced transfection [82]. At ultrasonic excitation levels 
below the cavitation threshold, lower shear forces can be induced, which can, for example, improve tissue growth by replicating in vivo conditions [83].

Ultrasonic 3D on-chip cell culture for a period of several days has been demonstrated [84]. The viability of acoustically trapped biological samples has also been explored, specifically for red blood cells [44] and zebrafish embryos [85] trapped for hours. In both studies, no compromise of biological function was observed, confirming that the samples retained their viability. However, in a recent study using a simple acoustophoretic lab-on-a-chip device [86], cell proliferation was unaffected, but an increase in metabolic activity was observed, suggesting that further research is required to properly understand the impact on biological viability or function in these devices. For a detailed review of the bio-effects of acoustic forces on cells, we refer readers to Ref. [79].

\section{[H1] MANIPULATION COMBINED WITH IMAGING}

The scope in applications of optical imaging has expanded immensely in recent years. At the macroscopic level, multimodal imaging is converging into 'optical biopsy' tools, and at the other end of the spectrum, super-resolution microscopy is pushing the limits down to the scale of a few nanometres. Contact-free confinement of a sample in a force field, as in optical and acoustic trapping or levitation, can create interesting scenarios for optical imaging. Trapping can isolate a sample well away from surfaces or other environmental effects, such as electrostatic or hydrodynamic influences. To make these benefits accessible, optical and acoustic trapping devices should be as compatible as possible with optical imaging.

Single-beam optical tweezers and dual-beam geometries are highly compatible with imaging approaches such as bright-field, phase-contrast or fluorescence microscopy. In particular, the marriage of optical tweezers, fluorescence microscopy and microfluidics has been very successful for biological studies with single-molecule manipulation, enabling single-molecule assays with excellent spatio-temporal resolution in tandem with the application of picoNewton forces. Systems that have been interrogated with this approach include molecular motors and protein-protein and protein-DNA interactions.

For exploring protein dynamics, optical tweezers were initially partnered with wide-field epi-fluorescent geometries and total internal reflection fluorescence microscopy. More recently, fluorescence microscopy (both one and two photon) have been used, even super- 
resolution imaging has been combined with single-beam traps [87]. Fluorescence imaging of trapped specimens can be challenging, as it is necessary to discriminate the fluorescence signal from the large background signals caused by the trap and the fluorescence excitation laser light [88].

Dual-beam optical traps have found application in microfluidic environments, accompanied by optics placed orthogonally to the trapping beam paths. Macro-tweezer mirror traps [17], which trap in the region between two focal spots typically separated by $100 \mu \mathrm{m}$, are particularly suitable for supporting imaging, as they possess a large field of view paired with a large working distance. The distance of the trap from the mirror can be selected over a wider range $(200-2,000 \mu \mathrm{m})$ than is typically possible with fibre-based traps, enabling an ultra-large optical manipulation volume of up to several $\mathrm{mm}^{3}$. The large and accessible volume facilitates additional optical imaging from the side with, for example, an inserted micro-prism. Furthermore, owing to a reduced risk of photodamage, macro-tweezers are advantageous for long-term imaging, including for bright-field imaging and Raman spectroscopy [89].

Like their optical counterparts, acoustic traps support optical imaging. The generally larger dimensions of acoustic traps makes access to the sample easier. Therefore, it is possible to make acoustic devices in which the piezoelectric actuators are arranged well away from the observation region, facilitating good optical access. An optically transparent acoustic $\mathrm{LiNbO}_{3}$ transducer with a transparent conductive indium tin oxide coating as the electrodes can be used to support high-NA imaging. However, the operating point has to be chosen with care, as the position of the objective lens may affect the acoustic resonances [90]. Transparent transducers have also been used to build miniature acoustic tweezers [91].

An auspicious application for acoustic and optical trapping is light sheet microscopy [92], which has come to the fore as a rapid volumetric imaging method in neuroscience and developmental biology. This technique has attracted interest owing to its decoupling of the illumination and imaging paths. This geometry leads to rapid 3D imaging with particularly low photodamage. Samples may be placed on coverslips, but several model organisms, such as zebrafish or embryos, would typically be immobilized by immersion in agarose gel. Although powerful, immobilization in a gel may restrict biological function and make it difficult for the introduction and removal of drugs or other buffers to the sample owing to limited diffusion. Various approaches have been demonstrated to address this issue. Magnetic beads 
have been introduced into samples, and the subsequent application of a tunable magnetic field enabled translational and rotational orientation of mouse and zebrafish embryos and larvae [93]. Both optical and acoustic traps can be used to immobilize samples in their native media without resorting to gels. Counter-propagating optical beams can be used, in this case using reflection from one side of the sample chamber itself, to hold small numbers of cells or microorganisms $[17,75]$.

Owing to the stronger forces and longer wavelengths, acoustic traps can accommodate larger samples. For example, a standing-wave acoustic trap chamber was recently used to immobilize zebrafish and other large embryos of up to several days post fertilization. Zebrafish are particularly attractive organisms for studying cardiovascular function owing to their optical transparency and their ability to regenerate tissue. The set-up made it possible to record quantitative data for the dynamic response of the heartbeat of zebrafish larvae to drugs that affect the cardiovascular system [94].

\section{[H1] OUTLOOK}

Optical and acoustic forces are setting the agenda for a number of emergent studies in biology and medicine. To a large extent, both fields are complementary and may be used powerfully in tandem. In the following, we discuss a few areas in which we expect significant developments along these lines.

\section{[H2] Mechanical probing in cell biology}

Mechanical factors are now thought to have an even greater role in cell biology than previously believed, with cell-cell interactions and mechanical interactions with the support structures having been shown to differ in 2D and 3D geometries [84, 95, 96]. Therefore, there are increasing efforts to mechanically probe cells and tissue. For example, cells deform and actively change when an external force is exerted, providing a powerful route for labelfree phenotyping and giving important insight into the development in organs and tissues in three dimensions and in vivo. In this respect, calibrated optical and acoustic forces can be applied at the lower end of the force range, with additional mechanical loading (for example, fluidic forces [97] or atomic-force-microscopy tips) used to increase the force 
exerted. A prime example of optical forces used in this way is the 'optical stretcher' [98]. Cell deformation along the axis of propagation of the light fields can be indicative of the cell state, enabling cells in different states to be distinguished. In applications, the optical stretcher is combined with a microfluidic system, which enables high-throughput measurements [99], or with acoustic prefocusing [100].

Acoustic forces can also be used to stretch biological samples, and acoustic versions of the optical stretcher have already been realized, including for cells and giant unilamellar vesicles [101-103]. For spherical (osmotically swollen) red blood cells, large deformations of up to $\sim 150 \%$ could be induced in optical stretchers. For these very large deformations, a substantial optical power of $\sim 500 \mathrm{~mW}$ was necessary [104]. The power level required to reach a deformation corresponding to an aspect ratio of 1.3-1.4 in a spherical red blood cell is four orders of magnitude lower in acoustic stretchers than in optical stretchers [103]. Moreover, the acoustic standing wave that induces the deformation can apply the stretching force to thousands of particles in parallel $[43,105]$, leading to the high cell throughput that is essential in applications such as mechanical phenotyping [106]. Another example of mechanical probing is the direct measurement of local viscosity in situ [107] using optically rotated birefringent microparticles that reach a terminal angular velocity when the optical torque [108] matches the rotational Stokes drag. Even inside a living animal, optical actuation is possible, as shown for otoliths in zebrafish larvae [109].

\section{[H2] Manipulation, patterning and the creation of spheroids and organoids}

A rapidly growing new application field for acoustofluidics is the handling and manipulation of spheroids and organoids. Three-dimensional acoustic force fields can be used to form arbitrary patterns using acoustic holograms [110, 111], or single traps can be used to efficiently fuse cells in suspension into spheroids [52, 84], and even to fuse these into larger organoids [112]. Acoustic waves have also proved useful for enhancing tissue formation, as it is possible to achieve sub-millimetre resolution patterning of fibres or cells for tissue engineering over a centimetre-scale region. Acoustic patterning can, for example, engineer muscle fibres from myoblasts with widths of $\sim 120-150 \mu \mathrm{m}$, which is still larger than in native tissue, but comparable to structures achievable with micropatterned hydrogels [31]. Moreover, it has already been demonstrated that acoustic standing waves with swept frequencies 
of $\sim 0.9 \mathrm{MHz}$ (in combination with microfluidic perfusion) facilitate the generation of 3D agglomerates of human chondrocytes and thus promote cartilage formation [113].

Acoustic and optical modalities have also been successfully applied to influence neuron growth. In optics, neuronal guiding is brought about by a microflow induced by a laserdriven optical micromachine consisting of birefringent microspheres placed close to the axonal growth cone that start spinning when exposed to circularly polarized light [114]. In the acoustic version, neurite growth in peripheral nerve regeneration was directed by acoustic patterning of Schwann cells in specific wave fields [30].

Apart from tissue engineering and studies on 3D cell assembly, acoustofluidics also lends itself to drug-screening studies, as cell aggregates and organoids are more accessible than in other cell-culture methods. In this context, much remains to be explored, in particular in combination with optical manipulation or optical and/or optogenetic targeting.

\section{[H2] Hybrid and in vivo approaches}

As discussed above, the advantages and limitations of optical and acoustic trapping are largely complementary in terms of size scales and the achievable and safely applicable forces. Therefore, combining the two modalities can forge a 'best of both worlds' approach for microscopic to mesoscopic biomedical investigations: acoustics can be used for the rough confinement and optics for the flexible and reconfigurable fine-tuning $[115,116]$.

The combination of acoustic and optical trapping also makes it possible to map out the acoustic force field using calibrated optical tweezers [90, 117-119]. This mapping is important because boundaries and optical imaging elements such as the objective lens have a strong influence on the acoustic resonances [61]. Although hybrid optical and acoustic systems were first implemented in 2011 (refs $[115,116]$ ), there is scope for new developments. We expect more compact, versatile and multi-purpose designs to emerge in the coming years that will make full use of the complimentary functions that optical and acoustic traps offer.

Both optical and acoustic trapping schemes could be further exploited for the in vivo manipulation of cells, an area that is in some respects still in its infancy. Acoustic forces could enable the localized release of therapeutic agents and offer prospects for neuroscience,

such as the contact-free study of embryo development. Optical tweezers have been used to manipulate individual red blood cells within subdermal capillaries in living mice, including 
clearing a blocked microvessel. There is substantial scope for researching live cell dynamics in animals [120]. Importantly, these studies will need increasingly sophisticated, yet robust, methods for introducing optical and acoustic forces in vivo. Both optical and acoustic forces can be tailored to have a gentle effect on biological organisms and to reduce any damage due to irradiation. We look ahead to more intricate studies in biomedicine using hybrid approaches in which the modalities optimally support each other.

Sound or light — which is right? The answer depends primarily on the application. Table 1 summarizes the suitability of optical and acoustic manipulation techniques in terms of the application requirements. Both modalities are powerful tools. Yet, combining the modalities certainly holds considerable potential, as either modality can mitigate the limitations of the other in terms of the accessible size-range or power-range for samples, or in terms of flexibility and imaging compatibility of the device.

[1] Ashkin, A. Acceleration and trapping of particles by radiation pressure. Physical Review Letters 24, 156-159 (1970).

[2] Ashkin, A., Dziedzic, J. M., Bjorkholm, J. \& Chu, S. Observation of a single-beam gradient force optical trap for dielectric particles. Optics Letters 11, 288-290 (1986).

[3] Jones, P. H., Maragò, O. M. \& Volpe, G. Optical tweezers: Principles and applications (Cambridge University Press, 2015).

[4] Sonnleitner, M., Ritsch-Marte, M. \& Ritsch, H. Optical forces, trapping and strain on extended dielectric objects. EPL (Europhysics Letters) 94, 34005 (2011).

[5] Sonnleitner, M., Ritsch-Marte, M. \& Ritsch, H. Optomechanical deformation and strain in elastic dielectrics. New Journal of Physics 14, 103011 (2012).

[6] Neto , P. M. \& Nussenzveig, H. Theory of optical tweezers. EPL (Europhysics Letters) 50, $702(2000)$.

[7] Stilgoe, A. B., Nieminen, T. A., Knöner, G., Heckenberg, N. R. \& Rubinsztein-Dunlop, H. The effect of Mie resonances on trapping in optical tweezers. Optics express 16, 15039-15051 (2008).

[8] Nieminen, T. A. et al. Optical tweezers computational toolbox. Journal of Optics A: Pure and Applied Optics 9, S196 (2007). 
[9] Nieminen, T. A., Loke, V. L., Stilgoe, A. B., Heckenberg, N. R. \& Rubinsztein-Dunlop, H. T-matrix method for modelling optical tweezers. Journal of Modern Optics 58, 528-544 (2011).

[10] Chu, S., Hollberg, L., Bjorkholm, J. E., Cable, A. \& Ashkin, A. Three-dimensional viscous confinement and cooling of atoms by resonance radiation pressure. Physical review letters 55, 48-51 (1985).

[11] Prentice, P. et al. Manipulation and filtration of low index particles with holographic laguerregaussian optical trap arrays. Optics Express 12, 593-600 (2004).

[12] Ruffner , D. B. \& Grier, D. G. Optical conveyors: a class of active tractor beams. Physical review letters 109, 163903 (2012).

[13] Dogariu, A., Sukhov, S. \& Sáenz, J. Optically induced 'negative forces'. Nature Photonics 7, 24-27 (2013).

[14] Thomas, J.-L. \& Marchiano, R. Pseudo angular momentum and topological charge conservation for nonlinear acoustical vortices. Phys. Rev. Lett. 91, 244302 (2003).

[15] Marston , P. L. \& Zhang, L. Radiation torques and forces in scattering from spheres and acoustical analogues. In Optical Trapping Applications, OMB5 (Optical Society of America, 2009).

[16] Curtis, J. E., Koss, B. A. \& Grier, D. G. Dynamic holographic optical tweezers. Optics Communications 207, 169-175 (2002).

[17] Thalhammer, G., Steiger, R., Bernet, S. \& Ritsch-Marte, M. Optical macro-tweezers: trapping of highly motile micro-organisms. Journal of Optics 13, 044024 (2011).

[18] Farré , A. \& Montes-Usategui, M. A force detection technique for single-beam optical traps based on direct measurement of light momentum changes. Optics express 18, 11955-11968 (2010).

[19] Thalhammer, G., Obmascher, L. \& Ritsch-Marte, M. Direct measurement of axial optical forces. Optics express 23, 6112-6129 (2015).

[20] Bustamante, C., Macosko, J. C. \& Wuite, G. J. Grabbing the cat by the tail: manipulating molecules one by one. Nature Reviews Molecular Cell Biology 1, 130-136 (2000).

[21] Carter, A. R., Seol, Y. \& Perkins, T. T. Precision surface-coupled optical-trapping assay with one-basepair resolution. Biophysical journal 96, 2926-2934 (2009).

[22] Pang , Y. \& Gordon, R. Optical trapping of a single protein. Nano Letters 12, 402-406 
(2011).

[23] Oddershede, L., Dreyer, J. K., Grego, S., Brown, S. \& Berg-Sørensen, K. The motion of a single molecule, the $\lambda$-receptor, in the bacterial outer membrane. Biophysical Journal 83, $3152-3161$ (2002).

[24] Montange, R., Bull, M. S., Shanblatt, E. R. \& Perkins, T. T. Optimizing bead size reduces errors in force measurements in optical traps. Optics Express 21, 39-48 (2012).

[25] Dyson, M., Woodward, B. \& Pond, J. Flow of red blood cells stopped by ultrasound. Nature 232, 572-573 (1971).

[26] Gorkov, L. P. On the forces acting on a small particle in an acoustic field in an ideal fluid. Sov. Phys. Dokl. 6, 773-775 (1962).

[27] Bruus, H. Acoustofluidics 7: The acoustic radiation force on small particles. Lab on a Chip 12, 1014-1021 (2012).

[28] Bruus, H. Acoustofluidics 2: Perturbation theory and ultrasound resonance modes. Lab on a Chip 12, 20-28 (2012).

[29] Laurell, T., Petersson, F. \& Nilsson, A. Chip integrated strategies for acoustic separation and manipulation of cells and particles. Chemical Society Reviews 36, 492-506 (2007).

[30] Gesellchen, F., Bernassau, A., Dejardin, T., Cumming, D. \& Riehle, M. Cell patterning with a heptagon acoustic tweezer-application in neurite guidance. Lab on a Chip 14, 2266-2275 (2014).

[31] Armstrong, J. P. et al. Engineering anisotropic muscle tissue using acoustic cell patterning. Advanced Materials 30, 1802649 (2018).

[32] Marzo, A. et al. Holographic acoustic elements for manipulation of levitated objects. Nature Communications 6, 8661 (2015).

[33] Guo, F. et al. Three-dimensional manipulation of single cells using surface acoustic waves. Proceedings of the National Academy of Sciences 113, 1522-1527 (2016).

[34] Baresch, D., Thomas, J.-L. \& Marchiano, R. Observation of a single-beam gradient force acoustical trap for elastic particles: acoustical tweezers. Physical Review Letters 116, 024301 (2016).

[35] Franklin, A., Marzo, A., Malkin, R. \& Drinkwater, B. Three-dimensional ultrasonic trapping of micro-particles in water with a simple and compact two-element transducer. Applied Physics Letters 111, 094101 (2017). 
[36] Ozcelik, A. et al. Acoustic tweezers for the life sciences. Nature Methods 15, 1021-1028 (2018).

[37] Allen, L., Beijersbergen, M. W., Spreeuw, R. \& Woerdman, J. Orbital angular momentum of light and the transformation of laguerre-gaussian laser modes. Physical Review A 45, 8185-8189 (1992).

[38] Yao , A. \& Padgett, M. Orbital angular momentum: origins, behavior and applications. Advances in Optics and Photonics 3, 161-204 (2011).

[39] Bliokh , K. Y. \& Nori, F. Spin and orbital angular momenta of acoustic beams. Physical Review B 99, 174310 (2019).

[40] Volke-Sepúlveda, K., Santillán, A. O. \& Boullosa, R. R. Transfer of angular momentum to matter from acoustical vortices in free space. Physical review letters 100, 024302 (2008).

[41] Marzo, A., Caleap, M. \& Drinkwater, B. W. Acoustic virtual vortices with tunable orbital angular momentum for trapping of mie particles. Physical Review Letters 120, 044301 (2018).

[42] Karlsen , J. T. \& Bruus, H. Forces acting on a small particle in an acoustical field in a thermoviscous fluid. Physical Review E 92, 043010 (2015).

[43] Silva, G. T., Lopes, J. H., Leão-Neto, J. P., Nichols, M. K. \& Drinkwater, B. W. Particle patterning by ultrasonic standing waves in a rectangular cavity. Physical Review Applied 11, 054044 (2019).

[44] Lam, K. H. et al. Multifunctional single beam acoustic tweezer for non-invasive cell/organism manipulation and tissue imaging. Scientific reports 6, 37554 (2016).

[45] Blake Jr, F. Bjerknes forces in stationary sound fields. The Journal of the Acoustical Society of America 21, 551-551 (1949).

[46] Burns, M. M., Fournier, J.-M. \& Golovchenko, J. A. Optical binding. Physical Review Letters 63, 1233-1236 (1989).

[47] Dholakia , K. \& Zemánek, P. Colloquium: Gripped by light: Optical binding. Reviews of Modern Physics 82, 1767-1791 (2010).

[48] Ku, A. et al. Acoustic enrichment of extracellular vesicles from biological fluids. Analytical chemistry 90, 8011-8019 (2018).

[49] Shi, J. et al. Acoustic tweezers: patterning cells and microparticles using standing surface acoustic waves (ssaw). Lab on a Chip 9, 2890-2895 (2009).

[50] Beyeler, F. et al. Monolithically fabricated microgripper with integrated force sensor for 
manipulating microobjects and biological cells aligned in an ultrasonic field. Journal of Microelectromechanical Systems 16, 7-15 (2007).

[51] Yeo, L. Y. \& Friend, J. R. Surface acoustic wave microfluidics. Annual review of fluid mechanics 46, 379-406 (2014).

[52] Chen, K. et al. Rapid formation of size-controllable multicellular spheroids via 3d acoustic tweezers. Lab on a Chip 16, 2636-2643 (2016).

[53] Bachman, H. et al. Low-frequency flexural wave based microparticle manipulation. Lab on a Chip 20, 1281-1289 (2020).

[54] Devendran, C., Gralinski, I. \& Neild, A. Separation of particles using acoustic streaming and radiation forces in an open microfluidic channel. Microfluidics and Nanofluidics 17, 879-890 (2014).

[55] Muller, P. B., Barnkob, R., Jensen, M. J. H. \& Bruus, H. A numerical study of microparticle acoustophoresis driven by acoustic radiation forces and streaming-induced drag forces. Lab on a Chip 12, 4617-4627 (2012).

[56] Bruus, H. Acoustofluidics 10: scaling laws in acoustophoresis. Lab on a Chip 12, 1578-1586 (2012)

[57] Barnkob, R., Augustsson, P., Laurell, T. \& Bruus, H. Acoustic radiation-and streaminginduced microparticle velocities determined by microparticle image velocimetry in an ultrasound symmetry plane. Physical Review E 86, 056307 (2012).

[58] Schmid, L., Weitz, D. A. \& Franke, T. Sorting drops and cells with acoustics: acoustic microfluidic fluorescence-activated cell sorter. Lab on a Chip 14, 3710-3718 (2014).

[59] Bruus, H. et al. Forthcoming lab on a chip tutorial series on acoustofluidics: Acoustofluidics - exploiting ultrasonic standing wave forces and acoustic streaming in microfluidic systems for cell and particle manipulation. Lab on a Chip 11, 3579-3580 (2011).

[60] Lenshof, A., Magnusson, C. \& Laurell, T. Acoustofluidics 8: Applications of acoustophoresis in continuous flow microsystems. Lab on a Chip 12, 1210-1223 (2012).

[61] Drinkwater, B. W. Dynamic-field devices for the ultrasonic manipulation of microparticles. Lab on a Chip 16, 2360-2375 (2016).

[62] Tian, Z. et al. Wave number-spiral acoustic tweezers for dynamic and reconfigurable manipulation of particles and cells. Science advances 5, eaau6062 (2019).

[63] Marzo , A. \& Drinkwater, B. W. Holographic acoustic tweezers. Proceedings of the National 
Academy of Sciences 116, 84-89 (2019).

[64] Alsteens, D., Tay, S. \& Müller, D. J. Toward high-throughput biomechanical phenotyping of single molecules. Nature Methods 12, 45-46 (2015).

[65] Abdelaziz , M. A. \& Grier, D. G. Acoustokinetics: Crafting force landscapes from sound waves. Physical Review Research 2, 013172 (2020).

[66] Démoré, C. E. et al. Acoustic tractor beam. Physical review letters 112, 174302 (2014).

[67] Maragò, O. M., Jones, P. H., Gucciardi, P. G., Volpe, G. \& Ferrari, A. C. Optical trapping and manipulation of nanostructures. Nature Nanotechnology 8, 807-819 (2013).

[68] Barredo, D., Lienhard, V., De Leseleuc, S., Lahaye, T. \& Browaeys, A. Synthetic threedimensional atomic structures assembled atom by atom. Nature 561, 79-82 (2018).

[69] Novotny, L., Bian, R. X. \& Xie, X. S. Theory of nanometric optical tweezers. Physical Review Letters 79, 645-648 (1997).

[70] Wang, K., Schonbrun, E., Steinvurzel, P. \& Crozier, K. B. Trapping and rotating nanoparticles using a plasmonic nano-tweezer with an integrated heat sink. Nature Communications 2, 469 (2011).

[71] Chen, M. et al. Observation of metal nanoparticles for acoustic manipulation. Advanced Science 4, 1600447 (2017).

[72] Wang, M. M. et al. Microfluidic sorting of mammalian cells by optical force switching. Nature Biotechnology 23, 83-87 (2005).

[73] MacDonald, M., Spalding, G. \& Dholakia, K. Microfluidic sorting in an optical lattice. Nature 426, 421-424 (2003).

[74] Ashkin, A., Dziedzic, J. M. \& Yamane, T. Optical trapping and manipulation of single cells using infrared laser beams. Nature 330, 769-771 (1987).

[75] Yang, Z., Piksarv, P., Ferrier, D. E., Gunn-Moore, F. J. \& Dholakia, K. Macro-optical trapping for sample confinement in light sheet microscopy. Biomedical Optics Express 6, 2778-2785 (2015).

[76] Neuman, K. C., Chadd, E. H., Liou, G. F., Bergman, K. \& Block, S. M. Characterization of photodamage to escherichia coli in optical traps. Biophysical journal 77, 2856-2863 (1999).

[77] Peterman, E. J., Gittes, F. \& Schmidt, C. F. Laser-induced heating in optical traps. Biophysical journal 84, 1308-1316 (2003).

[78] Ding, X. et al. On-chip manipulation of single microparticles, cells, and organisms using 
surface acoustic waves. Proceedings of the National Academy of Sciences 109, 11105-11109 (2012).

[79] Wiklund, M. Acoustofluidics 12: Biocompatibility and cell viability in microfluidic acoustic resonators. Lab on a Chip 12, 2018-2028 (2012).

[80] Iranmanesh, I. et al. Acoustic micro-vortexing of fluids, particles and cells in disposable microfluidic chips. Biomedical microdevices 18, 71 (2016).

[81] Fowlkes, J. B. \& Crum, L. A. Cavitation threshold measurements for microsecond length pulses of ultrasound. The Journal of the Acoustical Society of America 83, 2190-2201 (1988).

[82] Chen, Y. \& Lee, S. Manipulation of biological objects using acoustic bubbles: a review. Integrative and comparative biology 54, 959-968 (2014).

[83] Jonnalagadda, U. S. et al. Acoustically modulated biomechanical stimulation for human cartilage tissue engineering. Lab on a Chip 18, 473-485 (2018).

[84] Christakou, A. E., Ohlin, M., Önfelt, B. \& Wiklund, M. Ultrasonic three-dimensional on-chip cell culture for dynamic studies of tumor immune surveillance by natural killer cells. Lab on a Chip 15, 3222-3231 (2015).

[85] Sundvik, M., Nieminen, H. J., Salmi, A., Panula, P. \& Hæggström, E. Effects of acoustic levitation on the development of zebrafish, danio rerio, embryos. Scientific Reports 5, 13596 (2015).

[86] Devendran, C., Carthew, J., Frith, J. E. \& Neild, A. Cell adhesion, morphology, and metabolism variation via acoustic exposure within microfluidic cell handling systems. Advanced Science 6, 1902326 (2019).

[87] Heller, I. et al. Sted nanoscopy combined with optical tweezers reveals protein dynamics on densely covered dna. Nature Methods 10, 910-916 (2013).

[88] van Dijk, M., Kapitein, L., van Mameren, J., Schmidt, C. \& Peterman, E. J. G. Combining optical trapping and single-molecule fluorescence spectroscopy: Enhanced photobleaching of fluorophores. Physical Chemistry B 108, 6479-6484 (2004).

[89] Jess, P. et al. Dual beam fibre trap for raman microspectroscopy of single cells. Optics Express 14, 5779-5791 (2006).

[90] Thalhammer, G., McDougall, C., MacDonald, M. P. \& Ritsch-Marte, M. Acoustic force mapping in a hybrid acoustic-optical micromanipulation device supporting high resolution optical imaging. Lab on a Chip 16, 1523-1532 (2016). 
[91] Baudoin, M. et al. Folding a focalized acoustical vortex on a flat holographic transducer: miniaturized selective acoustical tweezers. Science Advances 5, eaav1967 (2019).

[92] Weber, M. \& Huisken, J. Light sheet microscopy for real-time developmental biology. Current opinion in genetics $\&$ development 21, 566-572 (2011).

[93] Berndt, F., Shah, G., Power, R. M., Brugués, J. \& Huisken, J. Dynamic and non-contact 3d sample rotation for microscopy. Nature Communications 9, 5025 (2018).

[94] Yang, Z. et al. Light sheet microscopy with acoustic sample confinement. Nature Communications 10, 669 (2019).

[95] Baker , B. M. \& Chen, C. S. Deconstructing the third dimension-how 3d culture microenvironments alter cellular cues. J Cell Sci 125, 3015-3024 (2012).

[96] Gómez-González, M., Latorre, E., Arroyo, M. \& Trepat, X. Measuring mechanical stress in living tissues. Nature Reviews Physics 2, 300-317 (2020).

[97] Berg-Sørensen, K. Optical two-beam traps in microfluidic systems. Japanese Journal of Applied Physics 55, 08RA01 (2016).

[98] Guck, J. et al. The optical stretcher: a novel laser tool to micromanipulate cells. Biophysical journal 81, 767-784 (2001).

[99] Lincoln, B., Wottawah, F., Schinkinger, S., Ebert, S. \& Guck, J. High-throughput rheological measurements with an optical stretcher. Methods in Cell Biology 83, 397-423 (2007).

[100] Nava, G. et al. All-silica microfluidic optical stretcher with acoustophoretic prefocusing. Microfluidics and Nanofluidics 19, 837-844 (2015).

[101] Mishra, P., Hill, M. \& Glynne-Jones, P. Deformation of red blood cells using acoustic radiation forces. Biomicrofluidics 8, 034109 (2014).

[102] Hwang, J. Y. et al. Cell deformation by single-beam acoustic trapping: a promising tool for measurements of cell mechanics. Scientific Reports 6, 27238 (2016).

[103] Silva, G. T. et al. Acoustic deformation for the extraction of mechanical properties of lipid vesicle populations. Physical Review E 99, 063002 (2019).

[104] Guck, J., Ananthakrishnan, R., Moon, T. J., Cunningham, C. \& Käs, J. Optical deformability of soft biological dielectrics. Physical Review Letters 84, 5451 (2000).

[105] Sitters, G. et al. Acoustic force spectroscopy. Nature Methods 12, 47-50 (2015).

[106] Urbanska, M., Rosendahl, P., Kraeter, M. \& Guck, J. High-throughput single-cell mechanical phenotyping with real-time deformability cytometry. In Methods in cell biology, vol. 147, 
175-198 (Elsevier, 2018).

[107] Bishop, A. I., Nieminen, T. A., Heckenberg, N. R. \& Rubinsztein-Dunlop, H. Optical microrheology using rotating laser-trapped particles. Physical Review Letters 92, 198104 (2004).

[108] Marston, P. L. \& Crichton, J. H. Radiation torque on a sphere caused by a circularly-polarized electromagnetic wave. Physical Review A 30, 2508-2516 (1984).

[109] Favre-Bulle, I. A., Stilgoe, A. B., Rubinsztein-Dunlop, H. \& Scott, E. K. Optical trapping of otoliths drives vestibular behaviours in larval zebrafish. Nature Communications 8, 630 (2017).

[110] Ma, Z. et al. Acoustic holographic cell patterning in a biocompatible hydrogel. Advanced Materials 32, 1904181 (2020).

[111] Melde, K., Mark, A. G., Qiu, T. \& Fischer, P. Holograms for acoustics. Nature 537, 518-522 (2016).

[112] Chen, P., Güven, S., Usta, O. B., Yarmush, M. L. \& Demirci, U. Biotunable acoustic node assembly of organoids. Advanced Healthcare Materials 4, 1937-1943 (2015).

[113] Li, S. et al. Application of an acoustofluidic perfusion bioreactor for cartilage tissue engineering. Lab on a Chip 14, 4475-4485 (2014).

[114] Wu, T. et al. A photon-driven micromotor can direct nerve fibre growth. Nature Photonics 6, 62-67 (2012).

[115] Thalhammer, G. et al. Combined acoustic and optical trapping. Biomedical Optics Express 2, 2859-2870 (2011).

[116] Glynne-Jones, P. \& Hill, M. Acoustofluidics 23: acoustic manipulation combined with other force fields. Lab on a Chip 13, 1003-1010 (2013).

[117] Bassindale, P., Phillips, D., Barnes, A. \& Drinkwater, B. Measurements of the force fields within an acoustic standing wave using holographic optical tweezers. Applied Physics Letters 104, 163504 (2014).

[118] Lamprecht, A., Lakämper, S., Baasch, T., Schaap, I. A. \& Dual, J. Imaging the positiondependent $3 \mathrm{~d}$ force on microbeads subjected to acoustic radiation forces and streaming. Lab on a Chip 16, 2682-2693 (2016).

[119] Memoli, G., Fury, C. R., Baxter, K. O., Gélat, P. N. \& Jones, P. H. Acoustic force measurements on polymer-coated microbubbles in a microfluidic device. The Journal of the Acoustical Society of America 141, 3364-3378 (2017). 
[120] Zhong, M.-C. Z., Wei, X.-B., Jin-Hua, Z., Wang, Z.-Q. \& Li, Y.-M. Trapping red blood cells in living animals using optical tweezers. Nature Communications 4, 1111 (2013). 


\section{ACKNOWLEDGEMENTS}

K.D. thanks the UK Engineering and Physical Sciences Research Council for funding (grant no. EP/P030017/1). B.D. gratefully acknowledges funding from the Wolfson Foundation and the Royal Society. M.R.M. gratefully acknowledges support from the Austrian Science Fund FWF (SFB-project F6806-N36) and helpful discussions with G. Thalhammer

and M. Kvåle Løvmo. The authors thank G. Bruce and P. Poulton for assistance with the figures.

\section{AUTHOR CONTRIBUTIONS}

The authors contributed equally to all aspects of the article.

\section{COMPETING INTERESTS}

The authors declare no competing interests.

\section{PUBLISHER'S NOTE}

Springer Nature remains neutral with regard to jurisdictional claims in published maps and institutional affiliations.

\section{PEER REVIEW INFORMATION}

Nature Reviews Physics thanks H. Rubinsztein-Dunlop, T. J. Huang and the other, anonymous, reviewer(s) for their contribution to the peer review of this work. 
FIG. 1. Optical and acoustic trapping fields. Schematic showing a vertical focused laser beam and a horizontal standing ultrasound wave both independently confining a particle.

\section{FIG. 2. Forces in optical tweezers.}

a) With the light propagating along the positive $z$ axis, a dielectric particle is drawn into the equilibrium position slightly above the focus of the laser beam. b,c) The total force $(F)$ is the sum of a conservative intensity gradient force (panel b) and a non-conservative radiation pressure force (panel c). $\lambda$, wavelength of the incident beam.

FIG. 3. Acoustic forces. Acoustic forces on small particles can be derived as gradients of the Gor'kov potential, but additional viscous forces due to acoustic streaming may be present. Adapted with permission from Ref.[33], PNAS.

\section{TOC BLURB}

Acoustic and optical waves can be used to exert non-contact forces on microscopic and mesoscopic objects. In this Technical Review, we compare and contrast the use of these modalities, or combinations thereof, in terms of sample manipulation and suitability for biomedical studies. 
TABLE I. Comparison of acoustic and optical manipulation techniques

\begin{tabular}{|c|c|c|c|c|c|}
\hline $\begin{array}{l}\text { Stable and non- } \\
\text { invasive confine- } \\
\text { ment }\end{array}$ & $\begin{array}{l}\text { Precision force } \\
\text { measurements }\end{array}$ & $\begin{array}{l}\text { 2D and } 3 \mathrm{D} \text { sam- } \\
\text { ple patterning }\end{array}$ & \begin{tabular}{|l|} 
Tissue engineer- \\
ing
\end{tabular} & $\begin{array}{l}\text { Compatibility } \\
\text { with imaging } \\
\text { and microflu- } \\
\text { idics }\end{array}$ & $\begin{array}{l}\text { Long-term mon- } \\
\text { itoring and bio- } \\
\text { compatibility }\end{array}$ \\
\hline \multicolumn{6}{|c|}{ Single-beam optical trapping (optical tweezers) } \\
\hline $\begin{array}{l}\text { Particle sizes in } \\
\text { the range } 10 \mathrm{~nm} \\
\text { to } 100 \mu \mathrm{m} \text {; forces } \\
\text { in the pico-Newton } \\
\text { regime; trapping } \\
\text { possible } r \text { inside } \\
\text { tissue or living } \\
\text { cells }\end{array}$ & $\begin{array}{l}\text { Various calibration } \\
\text { methods, enabling } \\
\text { forces and torques } \\
\text { to be measured in } \\
\text { situ }\end{array}$ & $\begin{array}{l}\text { Configurable 2D } \\
\text { and 3D arrays of } \\
\text { traps; } \quad \text { acousto- } \\
\text { optic } \quad \text { steering; } \\
\text { holographic tweez- } \\
\text { ers for 3D steering; } \\
\text { up to 100 trapped } \\
\text { particles }\end{array}$ & $\begin{array}{l}\text { Local applications } \\
\text { (cell infection); fu- } \\
\text { sion of individual } \\
\text { cells }\end{array}$ & $\begin{array}{l}\text { Compatible with } \\
\text { high-NA mi- } \\
\text { croscopy, most } \\
\text { imaging techniques } \\
\text { and microfluidics }\end{array}$ & $\begin{array}{l}\text { Largely unsuitable } \\
\text { for long-term mon- } \\
\text { itoring owing to } \\
\text { heating by absorp- } \\
\text { tion in sample and } \\
\text { solvent }\end{array}$ \\
\hline \multicolumn{6}{|c|}{ Dual-beam optical trapping } \\
\hline $\begin{array}{l}\text { Sample sizes of up } \\
\text { to } 100 \mu \mathrm{m} \text {; large, } \\
\text { accessible manip- } \\
\text { ulation volume; } \\
\text { long-term trapping }\end{array}$ & $\begin{array}{l}\text { Calibration can be } \\
\text { implemented; op- } \\
\text { tical stretching of } \\
\text { elastic specimens } \\
\text { possible }\end{array}$ & $\begin{array}{lr}\text { 1D trapping (along } \\
\text { optical axis); opti- } \\
\text { cal binding effects } \\
\text { between } & \text { trapped } \\
\text { particles } & \text { may } \\
\text { dominate } & \end{array}$ & $\begin{array}{l}\text { Unsuitable owing } \\
\text { to } 1 \mathrm{D} \text { confinement }\end{array}$ & $\begin{array}{l}\text { Facilitates imaging } \\
\text { from the side, an } \\
\text { orthogonal view } \\
\text { and cell tomog- } \\
\text { raphy; versatile } \\
\text { in microfluidic } \\
\text { chambers }\end{array}$ & $\begin{array}{l}\text { Absence of high- } \\
\text { intensity laser } \\
\text { spot greatly re- } \\
\text { duces negative side } \\
\text { effects }\end{array}$ \\
\hline \multicolumn{6}{|c|}{ Single-beam acoustic tweezers } \\
\hline $\begin{array}{l}\text { Strong forces; sam- } \\
\text { ples in air or water; } \\
\text { potential for exten- } \\
\text { sion to wide size } \\
\text { range }\end{array}$ & $\begin{array}{l}\text { Force } \\
\text { using gravibration } \\
\text { Stokes drag; bio- } \\
\text { logical selectivity } \\
\text { using functional- } \\
\text { ized microbeads }\end{array}$ & $\begin{array}{l}2 \mathrm{D} \text { and } 3 \mathrm{D} \text { trap- } \\
\text { ping using phased } \\
\text { arrays of piezo } \\
\text { transducers; } 3 \mathrm{D} \\
\text { only demonstrated } \\
\text { on objects } 50 \mu \mathrm{m} \text {; } \\
\text { manipulation of } \\
\text { clusters of about } \\
\text { five particles }\end{array}$ & $\begin{array}{l}\text { Unexplored but } \\
\text { with considerable } \\
\text { potential }\end{array}$ & $\begin{array}{lr}\text { Compatible } & \text { with } \\
\text { imaging } & \text { using } \\
\text { transparent } & \text { acous- } \\
\text { tic actuation } & \end{array}$ & $\begin{array}{l}\text { Cell manipulation } \\
\text { demonstrated but } \\
\text { no extensive re- } \\
\text { sults on biocom- } \\
\text { patibility }\end{array}$ \\
\hline \multicolumn{6}{|c|}{ Standing-wave acoustic tweezers } \\
\hline $\begin{array}{l}\text { Planar and spheri- } \\
\text { cal agglomerations } \\
\text { held for } 24 \mathrm{~h} \text { in } \\
3 \text {-axis levitators; } \\
\text { typical object size } \\
\text { range of } 1-500 \\
\mu \mathrm{m}\end{array}$ & 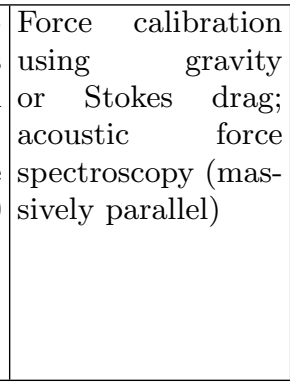 & \begin{tabular}{|lr} 
Formation & of $10^{3}$ \\
traps; & arbitrary \\
patterns & with \\
acoustic & holo- \\
grams; & particles \\
in traps & can be \\
moved or & trapped \\
en masse in 2D; 3D \\
lattice of & trapped \\
objects & \\
\end{tabular} & $\begin{array}{l}\text { Fusion of cells in } \\
\text { suspension into liv- } \\
\text { ing tissue }\end{array}$ & $\begin{array}{l}\text { Integration of } \\
\text { SAW devices with } \\
\text { microfluidics for } \\
\text { filtering or sorting; } \\
\text { throughput of }>10 \\
\text { ml min }^{-1}\end{array}$ & 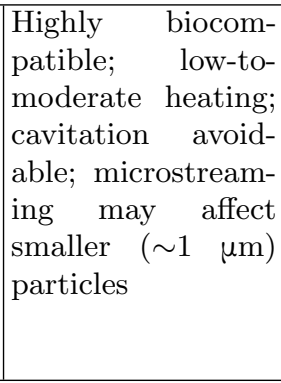 \\
\hline \multicolumn{6}{|c|}{ Combined sono-optical trapping } \\
\hline $\begin{array}{l}\text { Optical confine- } \\
\text { ment and acoustic } \\
\text { levitation can } \\
\text { be implemented } \\
\text { independently; } \\
\text { high-resolution op- } \\
\text { tical trapping and } \\
\text { strong acoustic } \\
\text { confinement }\end{array}$ & $\begin{array}{l}\text { Optical tweezers } \\
\text { can be used to } \\
\text { map out acoustic } \\
\text { force fields }\end{array}$ & \begin{tabular}{|lr} 
Various combina- \\
tions, such as a \\
steerable laser spot \\
with a $2 \mathrm{D}$ acoustic \\
standing & wave or a \\
$3 \mathrm{D}$ acoustic lattice; \\
optical redistri- \\
bution between \\
acoustic trapping \\
spots r
\end{tabular} & $\begin{array}{l}\text { Unexplored but } \\
\text { with considerable } \\
\text { potential }\end{array}$ & $\begin{array}{l}\text { Top view easily im- } \\
\text { plemented; sample } \\
\text { rotation for differ- } \\
\text { ent imaging direc- } \\
\text { tions; compatible } \\
\text { with microfluidics }\end{array}$ & $\begin{array}{l}\text { Highly biocompat- } \\
\text { ible; low to moder- } \\
\text { ate heating; cavita- } \\
\text { tion avoidable; mi- } \\
\text { crostreaming may } \\
\text { affect smaller }(\sim 1 \\
\mu \mathrm{m}) \text { particles }\end{array}$ \\
\hline
\end{tabular}

NA, numerical aperture; SAW, surface acoustic waves. The table gives an overview of the relative suitability of acoustic and optical manipulation techniques depending on the requirements of biophotonics applications, helping to answer the question of what method should be best used for the application at hand. 
FIG. 4. Acoustic and optical trapping regimes. Accessible trapping dimensions and force scales in optical and acoustic trapping with representative examples. $\lambda$, wavelength. 